PROCEEDINGS OF THE

AMERICAN MATHEMATICAL SOCIETY

Volume 139, Number 11, November 2011, Pages 4003-4016

S 0002-9939(2011)10818-9

Article electronically published on March 21, 2011

\title{
A FRACTIONAL HARDY-SOBOLEV-MAZ'YA INEQUALITY ON THE UPPER HALFSPACE
}

CRAIG A. SLOANE

(Communicated by Michael T. Lacey)

\begin{abstract}
We prove two Sobolev-type inequalities, which are then used to establish a fractional Hardy-Sobolev-Maz'ya inequality on the upper halfspace.
\end{abstract}

\section{INTRODUCTION}

The present work answers a question by Frank and Seiringer [9] concerning fractional Hardy-Sobolev-Maz'ya inequalities for the upper halfspace in the case $p=2$. Let

$$
\mathbb{R}_{+}^{n}=\left\{x=\left(x^{\prime}, x_{n}\right) \in \mathbb{R}^{n}: x^{\prime} \in \mathbb{R}^{n-1}, x_{n}>0\right\}
$$

be the upper halfspace, and let $\Omega$ be a domain in $\mathbb{R}^{n}$ with nonempty boundary. Then, there exists a fractional Hardy inequality on $\mathbb{R}_{+}^{n}$ which states that there exists $D_{n, p, \alpha}>0$ so that for all $f \in C_{c}\left(\mathbb{R}_{+}^{n}\right)$,

$$
\int_{\mathbb{R}_{+}^{n} \times \mathbb{R}_{+}^{n}} \frac{|f(x)-f(y)|^{p}}{|x-y|^{n+\alpha}} \mathrm{d} x \mathrm{~d} y \geq D_{n, p, \alpha} \int_{\mathbb{R}_{+}^{n}} \frac{|f(x)|^{p}}{x_{n}^{\alpha}} \mathrm{d} x,
$$

where $1 \leq p<\infty, 0<\alpha<p$, and $\alpha \neq 1$; see, e.g., [7]. Bogdan and Dyda found in [3] the sharp constant $D_{n, 2, \alpha}$ for the $p=2$ case. Later, the sharp constant $D_{n, p, \alpha}$ for general $p$ was found in [9]. Therein, the authors posed the question whether there existed a lower bound to the remainder for the inequality in (1.1) that is a positive multiple of the $L^{p^{*}}$-norm of $f$, where $p^{*}=n p /(n-\alpha)$ is the critical Sobolev exponent. Such an inequality would be a fractional analogue to the Hardy-SobolevMaz'ya inequality on the halfspace. Maz'ya [13] was the first to show the general result

$$
\int_{\mathbb{R}_{+}^{n}}|\nabla f(x)|^{2} \mathrm{~d} x-\frac{1}{4} \int_{\mathbb{R}_{+}^{n}} \frac{|f(x)|^{2}}{x_{n}^{2}} \mathrm{~d} x \geq C_{n}\left(\int_{\mathbb{R}_{+}^{n}}|f(x)|^{\frac{2 n}{n-2}} \mathrm{~d} x\right)^{\frac{n-2}{n}} .
$$

More recently the existence of minimizers for dimensions greater than or equal to 4 [14] and of the sharp constant for dimension 3 [4] has been established. Further improvements in the general case have been shown in 10 .

The following theorem for the sharp fractional Hardy inequality with remainder was proven by Frank and Seiringer in [9].

Received by the editors May 6, 2010 and, in revised form, September 28, 2010.

2010 Mathematics Subject Classification. Primary 26D10; Secondary 46E35.

This work was partially supported by NSF grant DMS 0901304.

(C)2011 American Mathematical Society 
Theorem 1.1. Let $n \geq 1,2 \leq p<\infty$, and $0<\alpha<p$ with $\alpha \neq 1$. Then for all $f \in C_{c}^{\infty}\left(\mathbb{R}_{+}^{n}\right)$,

$$
\begin{aligned}
\int_{\mathbb{R}_{+}^{n} \times \mathbb{R}_{+}^{n}} \frac{|f(x)-f(y)|^{p}}{|x-y|^{n+\alpha}} \mathrm{d} x \mathrm{~d} y-D_{n, p, \alpha} \int_{\mathbb{R}_{+}^{n}} \frac{|f(x)|^{p}}{x_{n}^{\alpha}} \mathrm{d} x \\
\quad \geq c_{p} \int_{\mathbb{R}_{+}^{n} \times \mathbb{R}_{+}^{n}} \frac{\left|x_{n}^{(1-\alpha) / p} f(x)-y_{n}^{(1-\alpha) / p} f(y)\right|^{p}}{|x-y|^{n+\alpha}} \frac{\mathrm{d} x}{x_{n}^{(1-\alpha) / 2}} \frac{\mathrm{d} y}{y_{n}^{(1-\alpha) / 2}},
\end{aligned}
$$

where $0<c_{p} \leq 1$ is given by

$$
c_{p}:=\min _{0<\tau<1 / 2}\left((1-\tau)^{p}-\tau^{p}+p \tau^{p-1}\right) .
$$

If $p=2$, then this is an equality with $c_{p}=1$.

For notational convenience, we write

$$
I_{\alpha, p}^{\Omega}(f)=\int_{\Omega \times \Omega} \frac{|f(x)-f(y)|^{p}}{|x-y|^{n+\alpha}} \mathrm{d} x \mathrm{~d} y
$$

and

$$
J_{\alpha, p}^{\Omega}(f)=\int_{\Omega \times \Omega} \frac{\left|x_{n}^{(1-\alpha) / p} f(x)-y_{n}^{(1-\alpha) / p} f(y)\right|^{p}}{|x-y|^{n+\alpha}} \frac{\mathrm{d} x}{x_{n}^{(1-\alpha) / 2}} \frac{\mathrm{d} y}{y_{n}^{(1-\alpha) / 2}} .
$$

Since we are primarily concerned with the case $p=2$, we further denote $I_{\alpha}^{\Omega}=I_{\alpha, 2}^{\Omega}$ and $J_{\alpha}^{\Omega}=J_{\alpha, 2}^{\Omega}$. Thus, for $p=2$, we can rewrite (1.2) as

$$
I_{\alpha}^{\mathbb{R}_{+}^{n}}(f)-D_{n, 2, \alpha} \int_{\mathbb{R}_{+}^{n}}|f(x)|^{2} x_{n}^{-\alpha} \mathrm{d} x=J_{\alpha}^{\mathbb{R}_{+}^{n}}(f) .
$$

The main result of this paper is the following fractional Hardy-Sobolev-Maz'ya inequality for $p=2$.

Theorem 1.2. Let $n \geq 2,1<\alpha<2$. There exists $a_{n, \alpha}>0$ so that

$$
\int_{\mathbb{R}_{+}^{n} \times \mathbb{R}_{+}^{n}} \frac{|f(x)-f(y)|^{2}}{|x-y|^{n+\alpha}} \mathrm{d} x \mathrm{~d} y-D_{n, 2, \alpha} \int_{\mathbb{R}_{+}^{n}} \frac{|f(x)|^{2}}{x_{n}^{\alpha}} \mathrm{d} x \geq a_{n, \alpha}\left(\int_{\mathbb{R}_{+}^{n}}|f(x)|^{2^{*}} \mathrm{~d} x\right)^{2 / 2^{*}},
$$

for all $f \in C_{c}^{\infty}\left(\mathbb{R}_{+}^{n}\right)$.

Alternatively, we write (1.4) as $J_{\alpha}^{\mathbb{R}_{+}^{n}}(f) \geq a_{n, \alpha}\|f\|_{2^{*}}^{2}$, where $\|\cdot\|_{p}$ refers to the $L^{p}$-norm with usual Lebesgue measure.

\section{Sobolev-TYPe INEQUALITIES}

Herein, we establish two Sobolev-type inequalities that we will need in the proof of Theorem 1.2. We prove each for the more general $p$-case. The first inequality we prove is for $I_{\alpha, p}^{\Omega}$ with respect to convex sets $\Omega$.

Theorem 2.1. Let $p \geq 2,1<\alpha<\min \{n, p\}$, and let $\Omega \subseteq \mathbb{R}^{n}$ be convex. Then, for all $f \in C_{c}^{\infty}(\Omega)$, there exists $c_{n, p, \alpha}>0$ so that

$$
I_{\alpha, p}^{\Omega}(f) \geq c_{n, p, \alpha}\|f\|_{p^{*}}^{p} .
$$


Proof. In [12, it is shown that

$$
I_{\alpha, p}^{\Omega}(f) \geq D_{n, p, \alpha} \int_{\Omega}|f(x)|^{p} d_{\Omega}(x)^{-\alpha} \mathrm{d} x
$$

for all $f \in C_{c}(\Omega)^{\infty}$, where $d_{\Omega}(x)=\operatorname{dist}(x, \partial \Omega)$. Further, there exists $S_{n, p, \alpha}$ (see, e.g., [1], Theorems $7.34,7.47)$ so that

$$
I_{\alpha, p}^{\mathbb{R}^{n}}(f) \geq S_{n, p, \alpha}\|f\|_{p^{*}}^{p},
$$

for all $f \in C_{c}^{\infty}\left(\mathbb{R}^{n}\right)$. Thus, if $B(x, r)$ is the ball of radius $r$ centered at $x$, then since

$$
\int_{\Omega^{c}}|x-y|^{-n-\alpha} \mathrm{d} y \leq \int_{B\left(x, d_{\Omega}(x)\right)^{c}}|x-y|^{-n-\alpha} \mathrm{d} y=\frac{1}{\alpha}\left|\mathbb{S}^{n-1}\right| d_{\Omega}(x)^{-\alpha},
$$

we have

$$
\begin{aligned}
S_{n, p, \alpha}\|f\|_{p^{*}}^{p} & \leq I_{\alpha, p}^{\mathbb{R}^{n}}(f) \\
& =I_{\alpha, p}^{\Omega}(f)+2 \int_{\Omega} \mathrm{d} x|f(x)|^{p} \int_{\Omega^{c}} \mathrm{~d} y|x-y|^{-n-\alpha} \\
& \leq I_{\alpha, p}^{\Omega}(f)+\frac{2}{\alpha}\left|\mathbb{S}^{n-1}\right| \int_{\Omega}|f(x)|^{p} d_{\Omega}(x)^{-\alpha} \mathrm{d} x \\
& \leq\left(1+\frac{2\left|\mathbb{S}^{n-1}\right|}{\alpha D_{n, p, \alpha}}\right) I_{\alpha, p}^{\Omega}(f),
\end{aligned}
$$

where $\left|\mathbb{S}^{n-1}\right|=\frac{2 \pi^{n / 2}}{\Gamma(n / 2)}$ is the surface area of the sphere of radius 1 in $\mathbb{R}^{n}$.

The next inequality is a weighted inequality for the term $J_{\alpha, p}^{\mathbb{R}_{+}^{n}}(f)$. As such, it can be viewed as a Hardy-Sobolev-Maz'ya type inequality. We leave the proof to the appendix.

Theorem 2.2. Let $p \geq 2,1<\alpha<\min \{n, p\}$. Then, there exists $d_{n, p, \alpha}>0$ so that

$$
J_{\alpha, p}^{\mathbb{R}_{+}^{n}}(f) \geq d_{n, p, \alpha}\left(\int_{\mathbb{R}_{+}^{n}}|f(x)|^{q} x_{n}^{-n+n q / p^{*}} \mathrm{~d} x\right)^{p / q},
$$

where $q=p\left(\frac{n+\frac{\alpha-1}{2}}{n-1}\right)$.

Since the remainder of the paper deals with the case $p=2$, we write $c_{n, \alpha}=c_{n, 2, \alpha}$ and $d_{n, \alpha}=d_{n, 2, \alpha}$.

In the next section, we will show that we can minimize over a certain class of functions that are decreasing, albeit not symmetrically. The crux of the proof of Theorem 1.2 is to decompose this function by truncation and to use these two inequalities to appropriately bound the $L^{2^{*}}$-norms of the resulting "upper" and "lower" functions.

\section{Class OF Minimizing FUnCtions}

In this section, we determine the properties of those functions that minimize our Rayleigh quotient

$$
\Phi_{\alpha}(f):=\frac{I_{\alpha}^{\mathbb{R}_{+}^{n}}(f)-D_{n, 2, \alpha} \int_{\mathbb{R}_{+}^{n}}|f(x)|^{2} x_{n}^{-\alpha} \mathrm{d} x}{\|f\|_{2^{*}}^{2}}=\frac{J_{\alpha}^{\mathbb{R}_{+}^{n}}(f)}{\|f\|_{2^{*}}^{2}} .
$$


3.1. Conformal transformation. Consider the conformal transformation $T$ : $B(\mathbf{0}, 1) \rightarrow \mathbb{R}_{+}^{n}$, where $\mathbf{0}$ is the origin in $\mathbb{R}^{n}$. If $\eta(\omega)=2 /\left(\left|\omega^{\prime}\right|^{2}+\left(\omega_{n}+1\right)^{2}\right)$, where $\omega=\left(\omega^{\prime}, \omega_{n}\right), \omega^{\prime} \in \mathbb{R}^{n-1}, \omega_{n} \in \mathbb{R}$, then

$$
T \omega=\left(\frac{2 \omega^{\prime}, 1-|\omega|^{2}}{\left|\omega^{\prime}\right|^{2}+\left(\omega_{n}+1\right)^{2}}\right)=\eta(\omega)\left(\omega^{\prime}, \frac{1-|\omega|^{2}}{2}\right) .
$$

The map $T$ can be extended as well from $\mathbb{R}^{n}$ onto $\mathbb{R}^{n}$, whereby the complement of $\mathbb{R}_{+}^{n}$ is mapped to the complement of the unit ball. Note that $T$ is an involution, and its Jacobian is $\eta(\omega)^{n}$ (see, e.g., Appendix in [6]). We define, depending on the value of $p$ in the fractional integral being used,

$$
\widetilde{f}(\omega):=\eta(\omega)^{n / p^{*}} f(T \omega),
$$

and since $\eta(T x)=1 / \eta(x)$, then

$$
f(x)=\eta(x)^{n / p^{*}} \tilde{f}(T x) .
$$

Thus, if supp $\tilde{f} \subseteq B(\mathbf{0}, 1)$, then supp $f \subseteq \mathbb{R}_{+}^{n}$. Indeed, for any $0<R<1$, if supp $\tilde{f} \subseteq B(\mathbf{0}, R)$, then supp $f \subseteq B^{R}$, where we define

$$
B^{R}:=\left\{\left(x^{\prime}, x_{n}\right) \in \mathbb{R}^{n}:\left|x^{\prime}\right|^{2}+\left(x_{n}-\frac{1+R^{2}}{1-R^{2}}\right)^{2} \leq\left(\frac{2 R}{1-R^{2}}\right)^{2}\right\},
$$

using $|T x|^{2}=\frac{\left|x^{\prime}\right|^{2}+\left(x_{n}-1\right)^{2}}{\left|x^{\prime}\right|^{2}+\left(x_{n}+1\right)^{2}}$.

We use these results to provide a new "ball" picture in which to consider our inequality and minimization problem. Among other things, we can also perform a rotation of $\tilde{f}$ on the ball. It turns out that repeated application of this rotation, along with a rearrangement along hyperplanes parallel to the boundary of $\mathbb{R}_{+}^{n}$, results in a limiting function that is radial in the ball picture and whose Rayleigh quotient is always smaller than that of the original.

3.2. Rearrangement on the upper halfspace. Using the transformation $T$, there is an interrelationship between those functions with support on the halfspace and those with support on the ball. This relationship will be used in this section to obtain a rearrangement for functions on the halfspace. We would like to use the spherically symmetric decreasing rearrangement, as it often assists in minimizing optimization problems on $\mathbb{R}^{n}$. However, such a tool is not useful for functions with support on the halfspace. Thus, at this time we must devise another.

Let $n \geq 2$ and $f \in L^{p^{*}}\left(\mathbb{R}_{+}^{n}\right)$. We consider two operations on nonnegative $f$. First, let $V f$ be the $(n-1)$-dimensional spherically symmetric decreasing rearrangement of $f$ in hyperplanes parallel to the boundary of $\mathbb{R}_{+}^{n}$. That is, for each $a>0$, we define $f_{a}\left(x^{\prime}\right)=f\left(x^{\prime}, a\right)$, where $x^{\prime} \in \mathbb{R}^{n-1}$. Then, $V f\left(x^{\prime}, a\right)=f_{a}^{*}\left(x^{\prime}\right)$, where $f_{a}^{*}$ represents the spherically symmetric decreasing rearrangement of $f_{a}$ in $\mathbb{R}^{n-1}$.

Next, let $U f$ be the transformation of $f$ obtained by a certain fixed rotation of $\tilde{f}$. In particular, using the rotation

$$
\mathbf{R}:\left(x_{1}, \ldots, x_{n-1}, x_{n}\right) \mapsto\left(x_{1}, \ldots, x_{n},-x_{n-1}\right), \quad x_{i} \in \mathbb{R}, i=1, \ldots, n,
$$

$U$ maps

$$
f(x) \mapsto \widetilde{f}(x) \mapsto \widetilde{f}(\mathbf{R} x) \mapsto \eta(x)^{n / p^{*}} \widetilde{f}(\mathbf{R} T x) .
$$

Note how the last transformation mimics the map $\widetilde{f} \mapsto f$. 
Now, define

$$
f_{k}:=(V U)^{k} f .
$$

Then, by Theorem 2.4 in [6], there exists $f^{\#} \in L^{p^{*}}\left(\mathbb{R}_{+}^{n}\right)$ with the following properties:

(1) $f^{\#}$ is nonnegative and spherically symmetric decreasing in hyperplanes $\left\{x \in \mathbb{R}^{n}: x_{n}=a\right\}, a>0$

(2) $f^{\#}$ is radially symmetric;

(3) $\|f\|_{p^{*}}=\left\|f^{\#}\right\|_{p^{*}}$; and

(4) $\lim _{k \rightarrow \infty} f_{k}=f^{\#}$ in $L^{p^{*}}\left(\mathbb{R}_{+}^{n}\right)$.

By passing to a subsequence, we can assume, without loss of generality, that $f_{k} \rightarrow$ $f^{\#}$ pointwise almost everywhere. We will refer to $f^{\#}$ as the pseudosymmetric halfspace rearrangment of $f$.

Using these properties, we can explicitly write $\widetilde{f} \#$ as the product of two radial functions in the ball picture: a specific, known spherically symmetric increasing function and a spherically symmetric decreasing function.

Theorem 3.1. Let $n \geq 2, f \in L^{p^{*}}\left(\mathbb{R}_{+}^{n}\right)$, where $f=f^{\#}$. Then, there exists a decreasing function $h:[0,1] \rightarrow[0, \infty]$, where $h(1)=0$, so that

$$
\widetilde{f}(w)=\left(\frac{2}{1-|w|^{2}}\right)^{n / p^{*}} h(|w|) .
$$

Proof. Let $x \in \mathbb{R}_{+}^{n}$ such that $x_{n}=1$, and, recalling that $T$ is an involution, let $w=T x$. Thus, if we restrict $T$ to the hyperplane

$$
H=\left\{\left(x^{\prime}, x_{n}\right) \in \mathbb{R}_{+}^{n}: x^{\prime} \in \mathbb{R}^{n-1}, x_{n}=1\right\},
$$

then the image, or stereographic projection, of $H$ under $T$ is the sphere

$$
S=\left\{w=\left(w^{\prime}, w_{n}\right): w^{\prime} \in \mathbb{R}^{n-1}, w_{n} \in \mathbb{R},\left|w^{\prime}\right|^{2}+\left(w_{n}+\frac{1}{2}\right)^{2}=\frac{1}{4}\right\}
$$

whose north and south poles pass through the origin and the point $(0, \ldots, 0,-1)$, respectively. Thus, for all $w \in S$, we have that $w_{n}=-|w|^{2}$, and $\eta(w)=2 /\left(1-|w|^{2}\right)$. Further,

$$
\frac{2}{1-|w|^{2}}=\eta(w)=\eta(T x)=\frac{1}{\eta(x)}=\frac{\left|x^{\prime}\right|^{2}+4}{2}
$$

whenever $x \in H$. Hence,

$$
\left|x^{\prime}\right|^{2}=\frac{4|w|^{2}}{1-|w|^{2}},
$$

for all $x \in H$, and, since $f$ is radial with respect to the $(n-1)$-dimensional hyperplane $H$, then, for all $w \in S$, we have

$$
f(T w)=f\left(x^{\prime}, 1\right)=f\left(\left|x^{\prime}\right|, 1\right)=f\left(\left|x^{\prime}\right|=\frac{2|w|}{\sqrt{1-|w|^{2}}}, 1\right) .
$$

Also, as $\widetilde{f}(w)=\eta(w)^{n / p^{*}} f(T w)$, then

$$
\widetilde{f}(w)=\left(\frac{2}{1-|w|^{2}}\right)^{n / p^{*}} f\left(\left|x^{\prime}\right|=\frac{2|w|}{\sqrt{1-|w|^{2}}}, 1\right)=\left(\frac{2}{1-|w|^{2}}\right)^{n / p^{*}} h(|w|),
$$


where $h(r)=f\left(\left|x^{\prime}\right|=\frac{2 r}{\sqrt{1-r^{2}}}, 1\right)$. Since $f$ is radially symmetric decreasing on $H$, $f \in L^{p^{*}}\left(\mathbb{R}_{+}^{n}\right)$, and

$$
\lim _{r \uparrow 1} \frac{2 r}{\sqrt{1-r^{2}}}=\infty,
$$

$h(r)$ must be a decreasing function, defined only on the interval $[0,1]$, such that $h(1)=0$.

Note that for each particular radius $R$ in the unit ball, the corresponding sphere $\partial B_{R}(\mathbf{0})$ intersects $S$. This radius then corresponds to a particular radius in $H$ as given by (3.2). But, on the ball, $\tilde{f}$ is a radial function, so if $w$ is any point in the unit ball, there exists some rotation $R_{w}$ and $w_{S} \in S$ so that $w=R_{w} w_{S}$. Therefore,

$$
\widetilde{f}(w)=\widetilde{f}\left(w_{S}\right)=\left(\frac{2}{1-\left|w_{S}\right|^{2}}\right)^{n / p^{*}} h\left(\left|w_{S}\right|\right)=\left(\frac{2}{1-|w|^{2}}\right)^{n / p^{*}} h(|w|),
$$

for all $w \in B$.

From this, it is a simple computation to show the following representation for $f^{\#}$ in the upper halfspace.

Corollary 3.2. Let $n \geq 2$ and $f \in L^{p^{*}}\left(\mathbb{R}_{+}^{n}\right)$. Then the pseudosymmetric halfspace rearrangement can be written as

$$
f^{\#}(x)=x_{n}^{-n / p^{*}} h(|T x|),
$$

where $h:[0,1] \rightarrow[0, \infty]$ is a decreasing function with $h(1)=0$.

Finally, we show that $\Phi_{\alpha}$ is nonincreasing under the map $f \mapsto f^{\#}$.

Theorem 3.3. Let $n \geq 2$, and let $f \in C_{c}^{\infty}\left(\mathbb{R}_{+}^{n}\right)$. Then, $\Phi_{\alpha}(f) \geq \Phi_{\alpha}\left(f^{\#}\right)$.

Proof. Recall the two operations $U, V$ on nonnegative $f$. Then, we define $F_{k}:=$ $(V U)^{k} f$ so that, passing to a subsequence if necessary, $F_{k} \rightarrow f^{\#}$ almost everywhere. Further, since $|f(x)-f(y)| \geq|| f|(x)-| f|(y)|$ implies $I_{\alpha}^{\mathbb{R}_{+}^{n}}(f) \geq I_{\alpha}^{\mathbb{R}_{+}^{n}}(|f|)$, we can assume $f$ is nonnegative.

We claim $\Phi_{\alpha}\left(F_{k}\right)$ is decreasing as $k \rightarrow \infty$. However, it is enough to show that $\Phi_{\alpha}(f) \geq \Phi_{\alpha}(V U f)$, since $F_{0}=f$. As was calculated in [3], there exists a constant $b_{n, \alpha}>0$, so we can write the remainder term as

$$
J_{\alpha}^{\mathbb{R}_{+}^{n}}(f)=I_{\alpha}^{\mathbb{R}^{n}}(f)-b_{n, \alpha} \int_{\mathbb{R}_{+}^{n}} \frac{|f(x)|^{2}}{x_{n}^{\alpha}} \mathrm{d} x .
$$

Hence, we can write

$$
\Phi_{\alpha}(f)=\frac{I_{\alpha}^{\mathbb{R}^{n}}(f)-b_{n, \alpha} \int_{\mathbb{R}_{+}^{n}} \frac{|f(x)|^{2}}{x_{n}^{\alpha}} \mathrm{d} x}{\|f\|_{2^{*}}^{2}} .
$$

Applying the transformation $U$ to $f$, we claim $\Phi_{\alpha}$ is invariant. Indeed, from Lemma 5.1, $I_{\alpha}^{\mathbb{R}^{n}}(f)=I_{\alpha}^{\mathbb{R}^{n}}(\tilde{f})$, the latter of which is invariant under rotations. Hence, $I_{\alpha}^{\mathbb{R}^{n}}(f)$ is invariant under $U$. Further, since

$$
\begin{aligned}
\int_{\mathbb{R}_{+}^{n}} f^{2}(x) x_{n}^{-\alpha} \mathrm{d} x & =\int_{B} f^{2}(T w)\left(\frac{1-|w|^{2}}{2} \eta(w)\right)^{-\alpha} \eta(w)^{n} \mathrm{~d} w \\
& =\int_{B}\left(\frac{2}{1-|w|^{2}}\right)^{\alpha} \widetilde{f}^{2}(w) \mathrm{d} w
\end{aligned}
$$


this is invariant under $U$ as well. It is clear the $L^{2^{*}}$-norm is also invariant under $U$. Hence, $\Phi_{\alpha}(f)=\Phi_{\alpha}(U f)$.

Next, we show that $\Phi_{\alpha}(U f) \geq \Phi_{\alpha}(V U f)$. From [8] and [1], it can be shown that $I_{\alpha}^{\mathbb{R}^{n}}(f)$ decreases under the spherically symmetric decreasing rearrangement. It is a simple application of the former, or modification of the latter, to show that $I_{\alpha}^{\mathbb{R}^{n}}(f)$ decreases under the rearrangement $V$ as well. Further, as the rearrangement under $V$ is only along hyperplanes parallel to the boundary of $\mathbb{R}_{+}^{n}$ (i.e., where $x_{n}$ is fixed), the integral

$$
\int_{\mathbb{R}_{+}^{n}} f^{2}(x) x_{n}^{-\alpha} \mathrm{d} x
$$

must be invariant under $V$. It is clear that the $L^{2^{*}}$-norm is invariant under $V$.

Therefore, applying Fatou's lemma and the properties of the pseudosymmetric halfspace rearrangement, we obtain

$$
\Phi_{\alpha}(f)=\frac{J_{\alpha}^{\mathbb{R}_{+}^{n}}(f)}{\|f\|_{2^{*}}^{2}}=\frac{J_{\alpha}^{\mathbb{R}_{+}^{n}}\left(F_{0}\right)}{\left\|f^{\#}\right\|_{2^{*}}^{2}} \geq \lim _{k \rightarrow \infty} \frac{J_{\alpha}^{\mathbb{R}_{+}^{n}}\left(F_{k}\right)}{\left\|f^{\#}\right\|_{2^{*}}^{2}} \geq \frac{J_{\alpha}^{\mathbb{R}_{+}^{n}}\left(f^{\#}\right)}{\left\|f^{\#}\right\|_{2^{*}}^{2}}=\Phi_{\alpha}\left(f^{\#}\right),
$$

as desired.

\section{Proof of the main Result}

Proof of Theorem 1.2. From Theorem 3.3 and Corollary 3.2, we can assume $f(x)=$ $x_{n}^{-n / p^{*}} h(|T x|)$, where $h(r)$ is a decreasing function on $[0,1]$ and $h(1)=0$. Then, $I_{\alpha}^{\mathbb{R}^{n}}(f)$ and $\|f\|_{2^{*}}^{2}$ remain finite. However, $f$ is no longer necessarily in $C_{c}^{\infty}\left(\mathbb{R}_{+}^{n}\right)$.

We decompose $h=h_{1}+h_{0}$ by truncation by fixing $R \in(0,1)$ so $h_{0}(r)=$ $\min \{h(r), h(R)\}$. Then, $f=f_{1}+f_{0}$, with the definitions $f_{1}, f_{0}$ following from the above. We claim there exist $c, d>0$, each dependent on $R, n$ and $\alpha$, such that

$$
J_{\alpha}^{\mathbb{R}_{+}^{n}}(f) \geq c\left\|f_{1}\right\|_{2^{*}}^{2}
$$

and

$$
J_{\alpha}^{\mathbb{R}_{+}^{n}}(f) \geq d\left\|f_{0}\right\|_{2^{*}}^{2} .
$$

Then, using the triangle and arithmetic-geometric mean inequalities, for all $0<$ $\lambda<1$, we obtain

$$
J_{\alpha}^{\mathbb{R}_{+}^{n}}(f) \geq \lambda c\left\|f_{1}\right\|_{2^{*}}^{2}+(1-\lambda) d\left\|f_{0}\right\|_{2^{*}}^{2} \geq \frac{1}{2} \min \{\lambda c,(1-\lambda) d\}\|f\|_{2^{*}}^{2} .
$$

Clearly, by fixing $\lambda, R$ not equal to zero or one, the constant is greater than zero. So, by taking the supremum over $\lambda$ and $R$, the result follows.

First, we prove (4.1). Note that supp $h_{1} \subseteq[0, R]$, so supp $f_{1} \subseteq B^{R}$, where $B^{R}$ is as in (3.1) above. Thus, for all $x, y \in B^{R}$,

$$
\begin{aligned}
\mid x_{n}^{(1-\alpha) / 2} & f_{1}(x)-\left.y_{n}^{(1-\alpha) / 2} f_{1}(y)\right|^{2} \\
& =\left|x_{n}^{\frac{1-n}{2}}(h(|T x|)-h(R))-y_{n}^{\frac{1-n}{2}}(h(|T y|)-h(R))\right|^{2} \\
& =\left|\left(x_{n}^{\frac{1-n}{2}} h(|T x|)-y_{n}^{\frac{1-n}{2}} h(|T y|)\right)+h(R)\left(y_{n}^{\frac{1-n}{2}}-x_{n}^{\frac{1-n}{2}}\right)\right|^{2} \\
& \leq 2\left|x_{n}^{(1-\alpha) / 2} f(x)-y_{n}^{(1-\alpha) / 2} f(y)\right|^{2}+2 h^{2}(R)\left|y_{n}^{\frac{1-n}{2}}-x_{n}^{\frac{1-n}{2}}\right|^{2} .
\end{aligned}
$$


It is easy to see that for any $0<R<1$,

$$
A_{1}=\int_{B^{R} \times B^{R}} \frac{\left|y_{n}^{(1-n) / 2}-x_{n}^{(1-n) / 2}\right|^{2}}{|x-y|^{n+\alpha}} x_{n}^{\frac{\alpha-1}{2}} y_{n^{\frac{\alpha-1}{2}}} \mathrm{~d} x \mathrm{~d} y<\infty,
$$

where $A_{1}$ is dependent on $R, n$ and $\alpha$. Thus, $J_{\alpha}^{B^{R}}(f)+A_{1} h^{2}(R) \geq \frac{1}{2} J_{\alpha}^{B^{R}}\left(f_{1}\right)$. We claim we can apply Theorem 2.1 to $x_{n}^{\frac{1-\alpha}{2}} f_{1}(x)$. Hence, if $x \in B^{R}$, then $\frac{1-R}{1+R} \leq$ $x_{n} \leq \frac{1+R}{1-R}$ and

$$
\begin{aligned}
J_{\alpha}^{B^{R}}\left(f_{1}\right) & \geq\left(\frac{1-R}{1+R}\right)^{\alpha-1} I_{\alpha}^{B^{R}}\left(x_{n}^{\frac{1-\alpha}{2}} f_{1}(x)\right) \\
& \geq c_{n, \alpha}\left(\frac{1-R}{1+R}\right)^{\alpha-1}\left(\int_{B^{R}} x_{n}^{\left(\frac{1-\alpha}{2}\right) 2^{*}}\left|f_{1}(x)\right|^{2^{*}} \mathrm{~d} x\right)^{2 / 2^{*}} \\
& \geq c_{n, \alpha}\left(\frac{1-R}{1+R}\right)^{2 \alpha-2}\left\|f_{1}\right\|_{2^{*}}^{2} .
\end{aligned}
$$

Using Theorem 2.2,

$$
J_{\alpha}^{\mathbb{R}_{+}^{n}}(f) \geq d_{n, \alpha}\left(\int_{\mathbb{R}_{+}^{n}}|h(|T x|)|^{q} x_{n}^{-n} \mathrm{~d} x\right)^{2 / q} \geq d_{n, \alpha} h^{2}(R)\left(\int_{B^{R}} x_{n}^{-n} \mathrm{~d} x\right)^{2 / q}=A_{2} h^{2}(R),
$$

where $A_{2}$ is also dependent on $R, n$ and $\alpha$. Therefore,

$$
\left(1+\frac{A_{1}}{A_{2}}\right) J_{\alpha}^{\mathbb{R}_{+}^{n}}(f) \geq J_{\alpha}^{B^{R}}(f)+A_{1} h^{2}(R) \geq \frac{1}{2} c_{n, \alpha}\left(\frac{1-R}{1+R}\right)^{2 \alpha-2}\left\|f_{1}\right\|_{2^{*}}^{2},
$$

which proves (4.1).

In establishing (4.2), we use the inequality $\frac{1}{2(n-1)} \leq \frac{\left(1-S^{2}\right)^{n-1}}{S^{n}} \int_{0}^{S} \frac{r^{n-1}}{\left(1-r^{2}\right)^{n}} \mathrm{~d} r$, $0<S<1$. Note that $h_{0}$ is constant on $[0, R]$, while it is decreasing to zero on $[R, 1]$. The following establishes how fast $h_{0}$ vanishes at 1 . From Theorem 2.2 ,

$$
\begin{aligned}
J_{\alpha}^{\mathbb{R}_{+}^{n}}(f) & \geq d_{n, \alpha}\left(\int_{\mathbb{R}_{+}^{n}}\left|h_{0}(|T x|)\right|^{q} x_{n}^{-n} \mathrm{~d} x\right)^{2 / q} \\
& =d_{n, \alpha}\left(2^{n}\left|\mathbb{S}^{n-1}\right| \int_{0}^{1} \frac{r^{n-1}}{\left(1-r^{2}\right)^{n}} h_{0}(r)^{q} \mathrm{~d} r\right)^{2 / q} \\
& \geq d_{n, \alpha} h_{0}(S)^{2}\left(\frac{2^{n-1}}{n-1}\left|\mathbb{S}^{n-1}\right|\right)^{2 / q}\left(\frac{S^{n}}{\left(1-S^{2}\right)^{n-1}}\right)^{2 / q}
\end{aligned}
$$

where $0<S<1$. Thus,

$$
h_{0}(r)^{2^{*}} \leq d_{n, \alpha}^{-2^{*} / 2}\left(\frac{2^{n-1}}{n-1}\left|\mathbb{S}^{n-1}\right|\right)^{-2^{*} / q}\left(\frac{\left(1-r^{2}\right)^{n-1}}{r^{n}}\right)^{2^{*} / q} J_{\alpha}^{\mathbb{R}_{+}^{n}}(f)^{2^{*} / 2}
$$


Then, we calculate

$$
\begin{aligned}
\left\|f_{0}\right\|_{2^{*}}^{2^{*}} & =2^{n}\left|\mathbb{S}^{n-1}\right| \int_{0}^{1} \frac{r^{n-1}}{\left(1-r^{2}\right)^{n}} h_{0}(r)^{2^{*}} \mathrm{~d} r \\
& =2^{n}\left|\mathbb{S}^{n-1}\right|\left(h_{0}(R)^{2^{*}} \int_{0}^{R} \frac{r^{n-1}}{\left(1-r^{2}\right)^{n}} \mathrm{~d} r+\int_{R}^{1} \frac{r^{n-1}}{\left(1-r^{2}\right)^{n}} h_{0}(r)^{2^{*}} \mathrm{~d} r\right) \\
\leq & 2^{n}\left|\mathbb{S}^{n-1}\right| d_{n, \alpha}^{-2^{*} / 2}\left(\frac{2^{n-1}}{n-1}\left|\mathbb{S}^{n-1}\right|\right)^{-2^{*} / q}\left(\left(\frac{\left(1-R^{2}\right)^{n-1}}{R^{n}}\right)^{2^{*} / q} \int_{0}^{R} \frac{r^{n-1}}{\left(1-r^{2}\right)^{n}} \mathrm{~d} r\right. \\
& \left.\quad+\int_{R}^{1} \frac{r^{n-1}}{\left(1-r^{2}\right)^{n}}\left(\frac{\left(1-r^{2}\right)^{n-1}}{r^{n}}\right)^{2^{*} / q} \mathrm{~d} r\right) J_{\alpha}^{\mathbb{R}_{+}^{n}}(f)^{2^{*} / 2} .
\end{aligned}
$$

As $2^{*}>q$, the claim follows.

Finally, we show that we can approximate $x_{n}^{\frac{1-\alpha}{2}} f_{1}(x)$ by functions in $C_{c}^{\infty}\left(B^{R}\right)$. Define

$$
g_{c}(x)=\max \left\{x_{n}^{\frac{1-\alpha}{2}} f_{1}(x)-c, 0\right\}
$$

almost everywhere. Then, by monotone convergence,

$$
I_{\alpha}^{B^{R}}\left(g_{c}\right) \rightarrow I_{\alpha}^{B^{R}}\left(x_{n}^{\frac{1-\alpha}{2}} f_{1}(x)\right), \quad\left\|g_{c}\right\|_{2^{*}}^{2} \rightarrow\left\|x_{n}^{\frac{1-\alpha}{2}} f_{1}(x)\right\|_{2^{*}}^{2},
$$

as $c \rightarrow 0$. Now, supp $g_{c}$ is a proper subset of $B^{R}$ and $g_{c} \in L^{2}\left(B^{R}\right)$, so $I_{\alpha}^{\mathbb{R}^{n}}\left(g_{c}\right)<\infty$ from (3.3). Denote

$$
\|\cdot\|_{W^{\alpha / 2,2}\left(\mathbb{R}^{n}\right)}=\sqrt{\|\cdot\|_{2}^{2}+I_{\alpha}^{\mathbb{R}^{n}}(\cdot)}
$$

and let $W_{0}^{\alpha / 2,2}\left(\mathbb{R}^{n}\right)$ be the completion of $C_{c}^{\infty}\left(\mathbb{R}^{n}\right)$ with respect to $\|\cdot\|_{W^{\alpha / 2,2}\left(\mathbb{R}^{n}\right)}$. Then it is known that

$$
W_{0}^{\alpha / 2,2}\left(\mathbb{R}^{n}\right)=W^{\alpha / 2,2}\left(\mathbb{R}^{n}\right)=\left\{u \in L^{2}\left(\mathbb{R}^{n}\right):\|u\|_{W^{\alpha / 2,2}\left(\mathbb{R}^{n}\right)}<\infty\right\} ;
$$

see, e.g., 1], 2]. Since supp $g_{c}$ is a proper subset of $B^{R}$, there exists a sequence $\left\{g_{c}^{j}\right\} \subset C_{c}^{\infty}\left(B^{R}\right)$ so that $\left\|g_{c}-g_{c}^{j}\right\|_{W^{\alpha / 2,2}\left(\mathbb{R}^{n}\right)} \rightarrow 0$ as $j \rightarrow \infty$. Hence, $I_{\alpha}^{B^{R}}\left(g_{c}^{j}\right) \rightarrow$ $I_{\alpha}^{B^{R}}\left(g_{c}\right)$ and $\left\|g_{c}^{j}\right\|_{2}^{2} \rightarrow\left\|g_{c}\right\|_{2}^{2}$ as $j \rightarrow \infty$.

\section{Appendix}

Proof of Theorem 2.2. We can assume that $f \geq 0$, since, by virtue of the triangle inequality,

$$
\left|x_{n}^{(1-\alpha) / p} f(x)-y_{n}^{(1-\alpha) / p} f(y)\right| \geq\left.|| x_{n}\right|^{(1-\alpha) / p}|f(x)|-\left|y_{n}\right|^{(1-\alpha) / p}|f(y)| \mid .
$$

Thus, we see that $J_{\alpha, p}^{\mathbb{R}_{+}^{n}}(f) \geq J_{\alpha, p}^{\mathbb{R}_{+}^{n}}(|f|)$.

We need a few preliminary results. Let $1_{\Omega}$ be the indicator function on the set $\Omega$. Then, for any $s \in \mathbb{R}$,

$$
\int_{0}^{\infty} s t^{-s-1} 1_{\{|x|<t\}} \mathrm{d} t=\int_{|x|}^{\infty} s t^{-s-1} \mathrm{~d} t=|x|^{-s} .
$$


The following is motivated by the Appendix in [5]. First, if $t \geq 0$, then $t^{p}=$ $p(p-1) \int_{0}^{\infty}(t-a)_{+} a^{p-2} \mathrm{~d} a$, where $t_{+}$is the positive part of $t$. Further note that $(|t|-a)_{+}=(t-a)_{+}+(-t-a)_{+}$, so, letting $a \geq 0$, we obtain

$$
\begin{aligned}
|g(x)-g(y)|^{p} & =p(p-1) \int_{0}^{\infty}(|g(x)-g(y)|-a)_{+} a^{p-2} \mathrm{~d} a \\
= & p(p-1) \int_{0}^{\infty}\left[(g(x)-g(y)-a)_{+}+(g(y)-g(x)-a)_{+}\right] a^{p-2} \mathrm{~d} a \\
= & p(p-1) \int_{0}^{\infty} \mathrm{d} a a^{p-2} \int_{0}^{\infty} \mathrm{d} b\left(1_{\{g(x)-a>b\}} 1_{\{g(y)<b\}}\right. \\
+ & \left.1_{\{g(y)-a>b\}} 1_{\{g(x)<b\}}\right),
\end{aligned}
$$

where the last equality is an identity in [5]. Now, define $g(x)=x_{n}^{\frac{1-\alpha}{p}} f(x)$. Then, letting $A=2 p(p-1)(n+\alpha)$ and using the results above,

$$
\begin{aligned}
& \int_{\mathbb{R}_{+}^{n}} \int_{\mathbb{R}_{+}^{n}} \frac{|g(x)-g(y)|^{p}}{|x-y|^{n+\alpha}} \frac{\mathrm{d} x}{x_{n}^{(1-\alpha) / 2}} \frac{\mathrm{d} y}{y_{n}^{(1-\alpha) / 2}} \\
& =\frac{1}{2} A \int_{\mathbb{R}_{+}^{n} \times \mathbb{R}_{+}^{n}} \frac{\mathrm{d} x}{x_{n}^{(1-\alpha) / 2}} \frac{\mathrm{d} y}{y_{n}^{(1-\alpha) / 2}} \int_{0}^{\infty} \frac{\mathrm{d} c}{c} c^{-n-\alpha} 1_{\{|x-y|<c\}} \int_{0}^{\infty} \mathrm{d} a a^{p-2} \int_{0}^{\infty} \mathrm{d} b \\
& \quad \times\left[1_{\{g(x)>a+b\}} 1_{\{g(y)<b\}}+1_{\{g(y)>a+b\}} 1_{\{g(x)<b\}}\right] \\
& =A \int_{\mathbb{R}_{+}^{n} \times \mathbb{R}_{+}^{n}} \frac{\mathrm{d} x}{x_{n}^{(1-\alpha) / 2}} \frac{\mathrm{d} y}{y_{n}^{(1-\alpha) / 2}} \int_{0}^{\infty} \frac{\mathrm{d} c}{c} c^{-n-\alpha} \int_{0}^{\infty} \mathrm{d} a a^{p-2} \int_{0}^{\infty} \mathrm{d} b 1_{\{|x-y|<c\}} \\
& \quad \times\left(1-1_{\{g(y) \geq b\}}\right) 1_{\{g(x)>a+b\}} .
\end{aligned}
$$

To simplify, let us make a few definitions. We write

$$
\lambda(a)=\int_{\mathbb{R}_{+}^{n}} 1_{\{g(x)>a\}} \frac{\mathrm{d} x}{x_{n}^{(1-\alpha) / 2}}
$$

and

$$
u(a, c)=\int_{\mathbb{R}_{+}^{n} \times \mathbb{R}_{+}^{n}} 1_{\{g(x)>a\}} 1_{\{|x-y|<c\}} \frac{\mathrm{d} x}{x_{n}^{(1-\alpha) / 2}} \frac{\mathrm{d} y}{y_{n}^{(1-\alpha) / 2}} .
$$

So, if $b \geq a$, then $u(a, c) \geq u(b, c)$ and $\lambda(a) \geq \lambda(b)$. Further, since $\alpha>1$, we obtain

$$
u(a, c) \geq \int_{\mathbb{R}_{+}^{n}} \frac{\mathrm{d} x}{x_{n}^{(1-\alpha) / 2}} 1_{\{g(x)>a\}} \int_{\mathbb{R}_{+}^{n}} \frac{\mathrm{d} y}{y_{n}^{(1-\alpha) / 2}} 1_{\{|y|<c\}}=D c^{n+\frac{\alpha-1}{2}} \lambda(a),
$$

where $D=\int_{\mathbb{R}_{+}^{n}} 1_{\{|y|<1\}} \frac{\mathrm{d} y}{y_{n}^{(1-\alpha) / 2}}$. Using Fubini,

$$
\begin{aligned}
& J_{\alpha, p}^{\mathbb{R}_{+}^{n}}= A \int_{0}^{\infty} \mathrm{d} a a^{p-2} \int_{0}^{\infty} \mathrm{d} b \int_{0}^{\infty} \frac{\mathrm{d} c}{c} c^{-n-\alpha} \int_{\mathbb{R}_{+}^{n} \times \mathbb{R}_{+}^{n}} \frac{\mathrm{d} x}{x_{n}^{(1-\alpha) / 2}} \frac{\mathrm{d} y}{y_{n}^{(1-\alpha) / 2}} \\
& \times\left(1_{\{|x-y|<c\}} 1_{\{g(x)>a+b\}}-1_{\{|x-y|<c\}} 1_{\{g(x)>a+b\}} 1_{\{g(y) \geq b\}}\right) \\
& \geq A \int_{0}^{\infty} \mathrm{d} a a^{p-2} \int_{0}^{\infty} \mathrm{d} b \int_{0}^{\infty} \frac{\mathrm{d} c}{c} c^{-n-\alpha}(u(a+b, c) \\
&\quad-\min \{u(a+b, c), u(b, c), \lambda(a+b) \lambda(b)\}) \\
& \geq \int_{0}^{\infty} \mathrm{d} a a^{p-2} \int_{0}^{\infty} \mathrm{d} b \int_{0}^{\infty} \frac{\mathrm{d} c}{c} c^{-n-\alpha} \lambda(a+b)\left(D c^{n+\frac{\alpha-1}{2}}-\lambda(b)\right)_{+} .
\end{aligned}
$$


From [11, Theorem 1.13, we can derive the formula $g^{q}(x)=\int_{0}^{\infty} q a^{q-1} 1_{\{g(x)>a\}} \mathrm{d} a$. As $g \geq 0$, we denote

$$
\|g\|_{q(\nu)}^{q}=\int_{\mathbb{R}_{+}^{n}}|g(x)|^{q} \frac{\mathrm{d} x}{x_{n}^{(1-\alpha) / 2}}=\int_{0}^{\infty} q a^{q-1} \lambda(a) \mathrm{d} a \geq \int_{0}^{b} q a^{q-1} \lambda(a) \mathrm{d} a=\lambda(b) b^{q} .
$$

Using the substitution $c=\left(\frac{\lambda(b)}{D}\right)^{\frac{2}{2 n+\alpha-1}} t$ and the identity $1-\frac{p}{q}=\frac{\alpha+1}{2 n+\alpha-1}$, we get

$$
\begin{aligned}
& \int_{\mathbb{R}_{+}^{n}} \int_{\mathbb{R}_{+}^{n}} \frac{|g(x)-g(y)|^{p}}{|x-y|^{n+\alpha}} \frac{\mathrm{d} x}{x_{n}^{(1-\alpha) / 2}} \frac{\mathrm{d} y}{y_{n}^{(1-\alpha) / 2}} \\
& \geq A D^{-\frac{2 n+2 \alpha}{2 n+\alpha-1}} \int_{0}^{\infty} \mathrm{d} a a^{p-2} \int_{0}^{\infty} \mathrm{d} b \lambda(a+b) \lambda(b)^{-\frac{\alpha+1}{2 n+\alpha-1}} \int_{1}^{\infty} \frac{\mathrm{d} t}{t} t^{-n-\alpha}\left(t^{n+\frac{\alpha-1}{2}}-1\right) \\
& \geq A \frac{2 n+\alpha-1}{(\alpha+1)(n+\alpha)} D^{-\frac{2 n+2 \alpha}{2 n+\alpha-1}}\|g\|_{q(\nu)}^{p-q} \int_{0}^{\infty} \mathrm{d} a a^{p-2} \int_{0}^{a} \mathrm{~d} b \lambda(a+b) b^{q-p} \\
& \geq \frac{A(2 n+\alpha-1) D^{-\frac{2 n+2 \alpha}{2 n+\alpha-1}}}{(\alpha+1)(n+\alpha)(q-p+1)}\|g\|_{q(\nu)}^{p-q} \int_{0}^{\infty} a^{q-1} \lambda(2 a) \mathrm{d} a \\
& =\frac{A(2 n+\alpha-1) D^{-\frac{2 n+2 \alpha}{2 n+\alpha-1}} 2^{-q}}{(\alpha+1)(n+\alpha)(q-p+1) q}\|g\|_{q(\nu)}^{p} \\
& =\frac{A(2 n+\alpha-1) D^{-\frac{2 n+2 \alpha}{2 n+\alpha-1}} 2^{-q}}{(\alpha+1)(n+\alpha)(q-p+1) q}\left(\int_{\mathbb{R}_{+}^{n}}|f(x)|^{q} x_{n}^{\frac{\alpha-1}{2}-q \frac{\alpha-1}{p}} \mathrm{~d} x\right)^{p / q} .
\end{aligned}
$$

Since $\frac{\alpha-1}{2}-q \frac{\alpha-1}{p}=-n+\frac{n q}{p^{*}}$, we are done.

Lemma 5.1. Let $n \geq 2,0<\alpha<2$, and $f \in C_{c}\left(\mathbb{R}^{n}\right)$. Then, $I_{\alpha}^{\mathbb{R}^{n}}(f)=I_{\alpha}^{\mathbb{R}^{n}}(\tilde{f})$, with the understanding that $I_{\alpha}^{\mathbb{R}^{n}}(\widetilde{f})=\infty$ if $I_{\alpha}^{\mathbb{R}^{n}}(f)=\infty$.

Proof. Let $f$ be a function with support in $\mathbb{R}^{n}$, and not necessarily in $\mathbb{R}_{+}^{n}$. Recall the transformation $T$, as discussed in Section 3.1, and the results from [6]. Then, we define $\widetilde{f}(x):=\eta(\omega)^{n / 2^{*}} f(T x)$, for all $x \in \mathbb{R}^{n}$.

We define the set

$$
A_{\epsilon}:=\left\{(x, y) \in \mathbb{R}^{n} \times \mathbb{R}^{n}: 1-\epsilon<\sqrt{\frac{\eta(y)}{\eta(x)}}<\frac{1}{1-\epsilon}\right\}
$$


Then, using the transformations $v=T x$ and $w=T y$, we obtain

$$
\begin{aligned}
& I_{\alpha}^{\mathbb{R}^{n}}(f)=\int_{\mathbb{R}^{n} \times \mathbb{R}^{n}} \frac{|f(v)-f(w)|^{2}}{|v-w|^{n+\alpha}} \mathrm{d} v \mathrm{~d} w \\
& =\int_{\mathbb{R}^{n} \times \mathbb{R}^{n}} \frac{|f(T x)-f(T y)|^{2}}{\left[\eta(x)|x-y|^{2} \eta(y)\right]^{(n+\alpha) / 2}}[\eta(x) \eta(y)]^{n} \mathrm{~d} x \mathrm{~d} y \\
& =\int_{\mathbb{R}^{n} \times \mathbb{R}^{n}} \frac{\left|\eta(x)^{(\alpha-n) / 2} \widetilde{f}(x)-\eta(y)^{(\alpha-n) / 2} \widetilde{f}(y)\right|^{2}}{|x-y|^{n+\alpha}}[\eta(x) \eta(y)]^{\frac{n-\alpha}{2}} \mathrm{~d} x \mathrm{~d} y \\
& =\lim _{\epsilon \rightarrow 0} \int_{\left(A_{\epsilon}\right)^{C}} \frac{\mathrm{d} x \mathrm{~d} y}{|x-y|^{n+\alpha}}\left[\left(\left(\frac{\eta(y)}{\eta(x)}\right)^{\frac{n-\alpha}{2}}-1\right) \widetilde{f}^{2}(x)\right. \\
& \left.+\left(\left(\frac{\eta(x)}{\eta(y)}\right)^{\frac{n-\alpha}{2}}-1\right) \widetilde{f}^{2}(y)+(\widetilde{f}(x)-\widetilde{f}(y))^{2}\right] \\
& =I_{\alpha}^{\mathbb{R}^{n}}(\widetilde{f})+2 \lim _{\epsilon \rightarrow 0} \int_{\left(A_{\epsilon}\right)^{C}} \frac{\widetilde{f}^{2}(x)}{|x-y|^{n+\alpha}}\left[\left(\frac{\eta(y)}{\eta(x)}\right)^{\frac{n-\alpha}{2}}-1\right] \mathrm{d} x \mathrm{~d} y .
\end{aligned}
$$

We show that the limit on the last line is zero for all $\epsilon>0$, thus establishing our result. We write $x=\left(x^{\prime}, x_{n}\right), x^{\prime} \in \mathbb{R}^{n-1}, x_{n} \in \mathbb{R}$. Then,

$$
\begin{aligned}
& \int_{\left(A_{\epsilon}\right)^{C}} \frac{\widetilde{f}^{2}(x)}{|x-y|^{n+\alpha}}\left[\left(\frac{\eta(y)}{\eta(x)}\right)^{\frac{n-\alpha}{2}}-1\right] \mathrm{d} x \mathrm{~d} y \\
& =\int_{\left(A_{\epsilon}\right)^{C}} \frac{\widetilde{f}^{2}\left(x^{\prime}, x_{n}\right)}{|x-y|^{n+\alpha}}\left[\left(\frac{\left|x^{\prime}\right|^{2}+\left(x_{n}+1\right)^{2}}{\left|y^{\prime}\right|^{2}+\left(y_{n}+1\right)^{2}}\right)^{\frac{n-\alpha}{2}}-1\right] \mathrm{d} x \mathrm{~d} y \\
& =\int_{\mathbb{R}^{n}} \mathrm{~d} x|x|^{n-\alpha} \widetilde{f}^{2}\left(x^{\prime}, x_{n}-1\right) \int_{\left\{y:(1-\epsilon)|x| \leq|y| \leq \frac{|x|}{1-\epsilon}\right\}^{C}} \mathrm{~d} y \frac{|y|^{\alpha-n}-|x|^{\alpha-n}}{|x-y|^{n+\alpha}} \\
& =\left|\mathbb{S}^{n-2}\right|\left(\int_{\mathbb{R}^{n}} \mathrm{~d} x \frac{\widetilde{f}^{2}\left(x^{\prime}, x_{n}-1\right)}{|x|^{\alpha}}\right) \\
& \quad \times\left(\int_{\left[1-\epsilon, \frac{1}{1-\epsilon}\right]^{C}} \mathrm{~d} t\left(t^{\alpha-1}-t^{n-1}\right) \int_{-1}^{1} \mathrm{~d} s \frac{\left(1-s^{2}\right)^{(n-3) / 2}}{\left(t^{2}+1-2 s t\right)^{(n+\alpha) / 2}}\right),
\end{aligned}
$$

where the complement in the last integral is with respect to the half line $[0, \infty)$. This product of integrals is zero as the left integral is finite, while the right integral is zero, for all $\epsilon>0$. Indeed, for the right integral, note that there is no singularity so long as $t \neq 1$. If we split the integral into $t$ above and below 1 , the latter must 
be finite, so the integral is finite if the sum is. We compute

$$
\begin{aligned}
& \int_{\left[1-\epsilon, \frac{1}{1-\epsilon}\right]} \mathrm{d} t\left(t^{\alpha-1}-t^{n-1}\right) \int_{-1}^{1} \mathrm{~d} s \frac{\left(1-s^{2}\right)^{(n-3) / 2}}{\left(t^{2}+1-2 s t\right)^{(n+\alpha) / 2}} \\
&=\int_{0}^{1-\epsilon} \mathrm{d} t\left(t^{\alpha-1}-t^{n-1}\right) \int_{-1}^{1} \mathrm{~d} s \frac{\left(1-s^{2}\right)^{(n-3) / 2}}{\left(t^{2}+1-2 s t\right)^{(n+\alpha) / 2}} \\
& \quad+\int_{\frac{1}{1-\epsilon}}^{\infty} \mathrm{d} t\left(t^{\alpha-1}-t^{n-1}\right) \int_{-1}^{1} \mathrm{~d} s \frac{\left(1-s^{2}\right)^{(n-3) / 2}}{\left(t^{2}+1-2 s t\right)^{(n+\alpha) / 2}} .
\end{aligned}
$$

In fact, the sum is zero, as

$$
\begin{aligned}
\int_{\frac{1}{1-\epsilon}}^{\infty} \mathrm{d} t\left(t^{\alpha-1}-t^{n-1}\right) & \int_{-1}^{1} \mathrm{~d} s \frac{\left(1-s^{2}\right)^{(n-3) / 2}}{\left(t^{2}+1-2 s t\right)^{(n+\alpha) / 2}} \\
& =\int_{0}^{1-\epsilon} \frac{\mathrm{d} t}{t^{2}}\left(t^{1-\alpha}-t^{1-n}\right) \int_{-1}^{1} \mathrm{~d} s \frac{\left(1-s^{2}\right)^{(n-3) / 2}}{\left(1 / t^{2}+1-2 s / t\right)^{(n+\alpha) / 2}} \\
& =\int_{0}^{1-\epsilon} \mathrm{d} t\left(t^{n-1}-t^{\alpha-1}\right) \int_{-1}^{1} \mathrm{~d} s \frac{\left(1-s^{2}\right)^{(n-3) / 2}}{\left(1+t^{2}-2 s t\right)^{(n+\alpha) / 2}} .
\end{aligned}
$$

Lastly, we consider the left integral. If we assume that $I_{\alpha}^{\mathbb{R}^{n}}(f)<\infty$, then, from 8 , there exists $c>0$ so that $\int_{\mathbb{R}^{n}} f^{2}(v)|v|^{-\alpha} \mathrm{d} v \leq c I_{\alpha}^{\mathbb{R}^{n}}(f)$. Hence, using $x=T v$,

$$
\begin{aligned}
\int_{\mathbb{R}^{n}} \widetilde{f}^{2}\left(x^{\prime}, x_{n}-1\right)|x|^{-\alpha} \mathrm{d} x & =2^{-\alpha / 2} \int_{\mathbb{R}^{n}} \widetilde{f}^{2}(T v) \eta(v)^{n-\alpha / 2} \mathrm{~d} v \\
& =\int_{\mathbb{R}^{n}} f^{2}\left(v^{\prime}, v_{n}-1\right)|v|^{-\alpha} \mathrm{d} v \\
& \leq c I_{\alpha}^{\mathbb{R}^{n}}(f)<\infty
\end{aligned}
$$

as desired. If $I_{\alpha}^{\mathbb{R}^{n}}(f)$ is not finite, then we need to consider whether

$$
\int_{\mathbb{R}^{n}} \widetilde{f}^{2}\left(x^{\prime}, x_{n}-1\right)|x|^{-\alpha} \mathrm{d} x<\infty .
$$

If (5.1) is true, then the result still holds. But, if (5.1) is not true, then since $\int_{\mathbb{R}^{n}} \widetilde{f}^{2}\left(x^{\prime}, x_{n}-1\right)|x|^{-\alpha} \mathrm{d} x \leq c I_{\alpha}^{\mathbb{R}^{n}}(\widetilde{f}), I_{\alpha}^{\mathbb{R}^{n}}(\widetilde{f})$ is also not finite.

\section{ACKNOWLEDGMENT}

The author is very thankful to Michael Loss for countless valuable discussions and especially for collaboration on Theorem 2.2 .

\section{REFERENCES}

1. R. Adams, J. Fournier, Sobolev Spaces. Second edition. Elsevier/Acad. Press, Amsterdam, 2003. MR2424078 (2009e:46025)

2. K. Bogdan, K. Burdzy, and Z.Q. Chen, Censored Stable Processes, Probab. Theory Related Fields 127, 89-152 (2003). MR2006232 (2004g:60068)

3. K. Bogdan, B. Dyda, The Best Constant in a Fractional Hardy Inequality, arXiv:0807.1825v1 [math.AP] (2008). 
4. R. Benguria, R. Frank, M. Loss, The Sharp Constant in the Hardy-Sobolev-Maz'ya Inequality in the Three Dimensional Upper Half-Space, Math. Res. Lett. 15, no. 4, 613-622 (2008). MR2424899 (2009j:46074)

5. E. Carlen, M. Loss, Extremals of Functionals with Competing Symmetries, J. Funct. Anal. 88 no. 2, 437-456 (1990). MR1038450 (91f:42021)

6. E. Carlen, M. Loss, On the Minimization of Symmetric Functionals. Special Issue Dedicated to Elliott H. Lieb. Rev. Math. Phys. 6, no. 5A, 1011-1032 (1994). MR1301364 (96k:58068)

7. B. Dyda, A Fractional Order Hardy Inequality, Ill. J. Math. 48, no. 2, 575-588 (2004). MR2085428 (2005f:26054)

8. R. Frank, R. Seiringer, Non-linear Ground State Representations and Sharp Hardy Inequalities, J. Funct. Anal. 255, 3407-3430 (2008). MR2469027 (2009k:46058)

9. R. Frank, R. Seiringer, Sharp Fractional Hardy Inequalities in Half-Spaces. In: Around the research of Vladimir Maz'ya, A. Laptev (ed.), 161-167, International Mathematical Series, 11, Springer, New York, 2010. MR2723817

10. S. Filippas, A. Tertikas, J. Tidblom, On the Structure of Hardy-Sobolev-Maz'ya Inequalities (English summary), J. Eur. Math. Soc. 11, no. 6, 1165-1185 (2009). MR2557132

11. E. Lieb, M. Loss. Analysis. Graduate Studies in Mathematics, vol. 14, Amer. Math. Society, Providence, RI, $2^{\text {nd }}$ edition, 2001. MR.1817225 (2001i:00001)

12. M. Loss, C. Sloane, Hardy Inequalities for Fractional Integrals on General Domains, J. Funct. Anal. 259, no. 6, 1369-1379 (2010). MR2659764

13. V. Mazya. Sobolev Spaces. Translated from the Russian by T. O. Shaposhnikova. Springer Series in Soviet Mathematics, Springer-Verlag, Berlin, 1985. MR.817985 (87g:46056)

14. A. Tertikas, K. Tintarev, On Existence of Minimizers for the Hardy-Sobolev-Maz'ya Inequality, Ann. Mat. Pura Appl. 186, 645-662 (2007). MR.2317783 (2008j:35069)

Department of Mathematics, Georgia Institute of Technology, Atlanta, Georgia $30332-0160$

E-mail address: csloane@math.gatech.edu 